\title{
Commentary \\ Recently published papers: Tracheostomy: why rather than when? Obesity: does it matter? And stroke: diagnosis, thrombosis and prognosis
}

\author{
Tim McCormick and Richard Venn
}

Worthing Hospital, Lyndhurst Road, Worthing BN11 2DH, UK

Corresponding author: Richard Venn, richard.venn@wash.nhs.uk

\begin{abstract}
Three studies explore the case for tracheostomies in the intensive care unit (ICU). Tracheostomies appear to have no effect on ICU survival, according to a prospective observational cohort study that used a propensity score. In obese patients, surgical tracheostomies were associated with an increased risk of complications, although these patients appeared to have a lower mortality in the ICU. A third study failed to show that tracheostomies reduced sedation requirements. MRI appears to be the investigation of choice for the diagnosis of acute stroke and thrombolysis is a safe and effective treatment for acute ischaemic strokes. Virtually all patients with a stroke may benefit from ongoing care in a stroke unit.
\end{abstract}

\section{Tracheostomy: why rather than when?}

The benefits of a tracheostomy are generally accepted reduced risk of laryngeal injury, weaning from intermittent positive pressure ventilation (IPPV), less sedation, improved patient comfort, communication and oral hygiene and, although risks exist, they are thankfully rare.

Early tracheostomy may improve survival, although its impact remains controversial (a controversy hopefully to be answered by the TracMan study [1]). The impact on morbidity/mortality is difficult to assess as patient factors and events in the intensive care unit (ICU) lead to bias - so called 'confounding in indication' - but by using a propensity score, Clec'h and colleagues [2] assessed the effect of a tracheostomy on mortality, allowing for bias. In this prospective observational cohort study, each patient with a tracheostomy was matched to mechanically ventilated patients without.

Of the 2,186 patients who received IPPV, 177 received tracheostomy (the majority surgical rather than percutaneous technique) and, after controlling for bias and confounding variables, tracheostomies were not associated with improved
ICU survival. There was no difference if the tracheostomy was placed before or after 7 days, although the median time for tracheostomy was 20 days (double the UK equivalent). In fact, tracheostomies appeared to be associated with increased post ICU mortality, especially in patients discharged with the tracheostomy remaining in situ.

Dr L'Her states in an editorial [3] that the patients were matched for the probabilities of getting a tracheostomy but it was possible that there were further factors that have not been controlled for - for example, numerous adverse events on the ICU, and so on. The increase in post ICU mortality in patients with a tracheostomy may reflect patients who were doing badly for a number of reasons and in whom the tracheostomy was left in place. It was, therefore, not surprising that mortality was worse in this group.

Given the variation in opinions on the use of tracheostomies, Nathens and colleagues [4] investigated the extent of variations in tracheostomy rates in trauma centres and what institutional or patient factors caused them.

In this analysis of a trauma databank, 4,146 patients underwent tracheostomy. The investigators found that tracheostomy rates varied widely (mean rate of 19.6 per 100 admissions; range 0 to 59) and the variation persisted after stratification by age, mechanism of injury and severity. There was also no association found with any institutional characteristics.

The variability in tracheostomy rates appeared to be driven by chance, physician preference and local culture opposed to medical indications, although it could reflect the preference of timing (timing was not recorded), where patients undergoing a late tracheostomy had the opportunity to recover before the procedure.

$\mathrm{BMI}=$ body mass index; $\mathrm{CT}=$ computerised tomography; ICU = intensive care unit; IPPV = intermittent positive pressure ventilation; $\mathrm{MRI}=$ magnetic resonance imaging; SIT-MOST = Safe Implementation of Thrombolysis in Stroke Monitoring Study. 
Dr Scales mentions in an editorial [5] that despite some data that may have helped clarify the variation being unavailable and there being no data on outcomes, the decision to perform a tracheostomy appears opinion based. When literature to support a procedure is lacking, we look to expert opinion - but what if this is contradictory?

One of the perceived benefits of a tracheostomy is a reduction in sedation requirements. In a retrospective analysis, Dr Veelo and colleagues [6] looked at sedation requirements in patients pre- and post-tracheostomy. Using a sedation protocol and a Sedation Intensive Care Score (SEDIC) patients were weaned from sedation. Of these, $62.4 \%$ required morphine before tracheostomy while $32.5 \%$ required morphine post-tracheostomy. Equivalent values were $44.4 \%$ versus $9.4 \%$ for midazolam and $34.2 \%$ versus $15.4 \%$ for propofol. There were no differences in prescribed sedatives in the two days before and after tracheostomy.

The authors conclude that sedation requirements were already in steep decline prior to tracheostomy and, although they commented that this was contrary to belief that tracheostomies reduce sedation requirements, it may just reflect the withdrawal of sedation as extubation is attempted. This does emphasize the importance of strict adherence to protocols or guidelines that aim to reduce sedation requirements in the ICU.

\section{Obesity: does it matter?}

Obesity, a preventable risk of morbidity and mortality, is increasing internationally. In New York, Drs Solh and Jaafar [7] looked at 455 critically ill patients who underwent a surgical trachesotomy and compared the incidence and severity of complications between 89 morbidly obese patients (body mass index $(\mathrm{BMI}) \geq 40$ ) and the remaining control group $(\mathrm{BMI}<40)$.

Complications were seen in $25 \%$ of morbidly obese patients (mortality 2\%) compared to $14 \%$ in the control group (mortality $0.6 \%$ ). The commonest serious complication was tracheostomy tube obstruction in obese patients and bleeding in the control group.

BMI, age and Charlson index (predictive index of mortality from co-morbid conditions) were found to be significant risks in univariate analysis, while only BMl was independently associated with increased risk of tracheostomy-related complications.

Interestingly, obesity was not associated with a worse outcome in a prospective observational cohort study evaluating the effects of BMI on 12 month survival in critically ill patients [8].

Mortality did, however, increase with low albumin, advanced age and comorbid disease and the authors postulate that reasons for their contrary findings centre around different end points or bias - perhaps medical problems associated with obesity rather than obesity per se are risk factors. They suggest a high BMI may provide a nutritional reserve in times of stress but admit that although BMI is widely used, it does not distinguish between fat and muscle - a waist to hip ratio may be a more accurate measure.

\section{Stroke: diagnosis, thrombosis and prognosis}

Dr Chalela and colleagues [9] report the superior results of magnetic resonance imaging (MRI) over computerised tomography $(\mathrm{CT})$ for detection of acute stroke.

In this single centre, prospective blind comparison of noncontrast CT and MRI, MRI detected acute stroke (ischaemic or haemorrhagic) and chronic haemorrhage more frequently than CT. Detection of acute intracerebral bleed was similar for both investigations. MRI had a sensitivity of $83 \%$ while CT scored $16 \%$ for detection of acute ischaemic stroke.

The diagnostic accuracy of MRI was the same for scans within the first 3 hours from onset of symptoms as it was for later scans, which is relevant for thrombolysis. However Donnan and Dewey [10] point out that $11 \%$ of patients were unable to undergo MRI and the practicalities of intubated patients need to be considered.

Alteplase (tPA) has been granted a license for use in the US and Canada for ischaemic stroke. Administration within a 3 hour window from symptom onset has previously been shown to be safe and effective - treated patients being at least $30 \%$ more likely to have little or no disability.

Prior to gaining a license in Europe a large safety study was required, and thus the Safe Implementation of Thrombolysis in Stroke Monitoring Study (SIT-MOST) was carried out [11]. In this prospective open monitored observational study, primary outcomes were symptomatic intracerebral haemorrhage and death within three months.

Data from SIT-MOST suggest there was a noticeable reduction in 3 month mortality (mortality 11.3\%) and this reduction was seen in both inexperienced and experienced centres. The rate of symptomatic intracranial haemorrhage was low (1.7\%).

This study confirmed the safety profile and efficacy of alteplase. Although this may be the gold standard of acute stroke care, it will require significant expansion of current services. Patients were given thrombolysis on average 68 minutes after coming through the door and to match this would require substantial effort and investment. Perhaps these efforts would be better spent by concentrating on prevention.

Further experimental treatments for stroke are explored in an excellent review in the Lancet [12]. 
Do stroke units work? Evidence from randomized controlled small single centre trials suggests that stroke units are beneficial. Dr Candelise and colleagues [13] investigated whether acute admission ( $<48$ hours) to a stroke unit affected outcome of patients compared to conventional ward care in an observational follow up study of 11,572 acute stroke patients from 260 hospitals. Of these, 4,936 went to a stroke unit while 6,636 went to a conventional ward.

Compared with conventional ward based care, the stroke units were associated with reduced mortality and disability these benefits persisted across all age ranges and clinical characteristics (except unconsciousness), adding to the overall impression that stroke units are of benefit and no patient should be discriminated against.

\section{Conclusions}

There remain many unanswered questions with regards to tracheostomies, notably optimum timing and who benefits? A surgical tracheostomy is associated with a higher mortality in morbidly obese patients but this patient group may actually do better in the ICU.

Acute stroke patients should be offered an MRI and subsequent thrombolysis where appropriate and continuation of care should be in stroke units and, although this represents 'best' treatment, is this the best use of resources and efforts?

\section{Competing interests}

The authors declare that they have no competing interests.

\section{References}

1. TracMan [www.tracman.org.uk]

2. Clec'h C, Alberti C, Vincent F, Garrouste-Orgeas M, de Lassence A, Toledano D, Azoulay E, Adrie C, Jamali S, Zaccaria I, et al.: Tracheostomy does not improve the outcome of patients requiring prolonged mechanical ventilation: A propensity analysis. Crit Care Med 2007, 35:132-138.

3. L'Her E: Tracheostomy: May the truth be out there. Crit Care Med 2007, 35:309-310.

4. Nathens A, Rivara F, Mack C, Rubenfeld G, Wang J, Jurkovich G, Maier V: Variations in rates of tracheostomy in the critically ill trauma patient. Crit Care Med 2007, 34:2919-2924

5. Scales D, Ferguson N: Tracheostomy: It is time to move from art to science. Crit Care Med 2006, 34:3039-3040

6. Veelo D, Dongelmans D, Binnekade J, Korevaar J, Vroom M, Schultz M: Tracheotomy does not affect reducing sedation requirements of patients in intensive care - a retrospective study. Crit Care 2006, 10:R99.

7. Solh A, Jaafar W: A comparative study of the complications of surgical tracheostomy in morbidly obese critically ill patients. Crit Care 2007, 11:R3.

8. Peake S, Moran J, Ghelani D, Lloyd A, Walker M: The effect of obesity on 12-month survival following admission to intensive care: a prospective study. Crit Care Med 2006, 34:2929-2939.

9. Chalela J, Kidwell C, Nentwich L, Luby M, Butman J, Demchuk A, Hill M, Patronas N, Latour L, Warach S: Magnetic resonance imaging and computerised tomography in emergency assessment of patients with suspected acute stroke: a prospective comparison. Lancet 2007, 369 :293-298.

10. Donnan G, Dewey H: MRI and stroke: why has it taken so long? Lancet 2007, 369:252-254.

11. Wahlgren N, Ahmed N, Davalos A, Ford G, Grond W, Hacke W, Hennerici M, Kaste M, Kuelkens S, Larrue V, et al.: Thrombolysis with alteplase for acute ischaemic stroke in the Safe Implementation of Thrombolysis in Stroke Monitoring Study (SITMOST): an observational study. Lancet 2007, 369:275-282.

12. Sacco R, Chong J, Prabhakaran S, Elkind M: Experimental treatments for acute ischaemic stroke. Lancet 2007, 369:331-341.

13. Candelise L, Gattinoni M, Bersano A, Micieli G, Sterzi R, Morabito A: Stroke-unit care for acute stroke patients. Lancet 2007, 369:299-305 